\title{
Syndromic Surveillance at the New York State Veterinary Diagnostic Laboratory
}

\author{
Kylius Wilkins* ${ }^{* 1}$, Bruce Akey2, ${ }^{3}$, Belinda Thompson ${ }^{2,3}$ and Daryl Nydam ${ }^{3}$ \\ ${ }^{1}$ Veterinary Services, USDA APHIS, Ames, IA, USA; ${ }^{2} \mathrm{NYSVDL}$, Ithaca, NY, USA; ${ }^{3}$ Cornell University, Ithaca, NY, USA
}

\section{Objective}

To assess the use and utility of a syndrome check list on the general submission form of a high volume veterinary diagnostic laboratory, and compare to the results of a 2009 pilot study.

\section{Introduction}

The New York State Veterinary Diagnostic Laboratory (NYSVDL) receives more than 100,000 diagnostic submissions a year that are not currently used in any formal syndromic surveillance system. In 2009 , a pilot study of syndrome classification schemes was undertaken and in 2011 a new general submission form was adopted, which includes a check list of syndromes, as part of the clinical history.

Monitoring submissions to a veterinary diagnostic laboratory for increases in certain test requests is an established method of syndromic surveillance $(1,2)$. The new general submission form allows for clinician selected syndromes to be monitored in addition to test request.

\section{Methods}

We selected 420 "contract cases" from all submissions for bovines since the implementation of the new form, May 2011, though February 2012. Submissions were reviewed for use of the new form, use of the syndrome check list and tests requested. Test requests were assigned syndromes, if possible, to allow for comparison with the clinician selected syndromes.

The selection of cases was weighted towards the end of the period as use of the new form increased with time and to a lesser extent toward the beginning of the period in an attempt to find any early use of the form. "Contract case" refers to New York State Department of Agriculture and Markets subsidization of agricultural animal and herd health testing done under specific guidelines. The benefit of "contract cases" is the guidelines require a complete clinical history, which should include selection of syndromes. Finally, selection was limited bovine submissions as was done in the pilot study.

\section{Results}

$16 \%(69 / 420)$ of submissions used the new form and of these 23 selected syndromes. As was seen in the pilot study the most commonly occurring syndrome in the clinical history was "Gastrointestinal/Diarrhea" 56\% (13/23). The next most common syndromes were "Respiratory" (7/23), "Sudden Death" (6/23) and "Fever" (4/23).

Syndromes assigned based on test request followed a similar pattern with "Gastrointestinal/Diarrhea" (166/254) and "Respiratory" $(52 / 254)$ best represented. An important difference was the syndromes "Sudden Death" and "Fever", which were never assigned to a test request. These syndromes represent a new source of information for surveillance.
These results fit well with the pilot study which found the clinical history was typically incomplete but contained additional information for syndromic surveillance that was not available from monitoring the test request alone and that monitoring syndromes or test requests alone would provide incomplete information nearly a third of the time.

\section{Conclusions}

We found monitoring syndromes, in addition to test requests, to be useful and necessary for completeness. Monitoring clinical history provided additional information not available from test requests alone. We recommend the syndromes "Sudden Death" and "Fever" be monitored as these syndromes always provided additional information not available in test requests. Other syndromes that provide new information should be investigated across species and in various clinical scenarios. Accumulating baseline data for all syndromes is recommended to create more accurate models for syndromic surveillance and improve data retrieval for retrospective studies. Despite poor use of the new general submission form and the syndrome check boxes, future compliance is likely to improve significantly with the implementation of online submission and thanks to the continuous training and consultation provided by the NYSVDL staff.

\section{Keywords}

Syndromic Surveillance; Veterinary Diagnostic Laboratory; Veterinary Surveillance

\section{Acknowledgments}

We would like to thank Dr. Robert Gilmour and the Cornell University Veterinary Investigator Program for supporting this research.

\section{References}

1. Shaffer, L., Funk, J., Rajala-Schultz, P., Wallstrom, G., Wittum, T. Wagner, M., Saville, W. (2007) Early Outbreak Detection Using an Automated Data Feed of Test Orders from a Veterinary Diagnostic Laboratory. In: D. Zeng et al. (Ed), BioSurveillance 2007, pp. 1-10

2. Glickman, L.T., Moore, G.E., Glickman, N.E., Caldanaro, R.J., Aucoin, D., Lewis, H.B. (2006) Purdue University-Banfield National Companion Animal Surveillance Program for Emerging and Zoonotic Diseases Vector-Borne and Zoonotic Diseases. 6(1), 14-23.

3. Dorea, F.C., Sanchez, J., Crawford, W.R. (2011) Veterinary syndromic surveillance: Current initiatives and potential for development. Preventive Veterinary Medicine 101, 1-17

*Kylius Wilkins

E-mail: kmw97@cornell.edu 